\title{
Psendotumor der Iris bei einem Kinde.
}

\author{
Von \\ Dr. Siegmund Ginsberg und Dr. Paul Cohn \\ in Berlin.
}

Mit Taf. XIII, Kig. 1 u. 2.

Krankengeschichte.

(Dr. C.) Am 11. III. 1911 stellte sich in der „Kinder-Augenheilanstalt" der $\left.4_{1}^{1}\right|_{2}$ jährige Knabe Curt M. vor. Nach Angabe der Mutter hatte das Kind mit 11 Monaten eine schwere Masernerkrankung mit anschliessender äusserst schwerer und langwieriger Entzündung des rechten Auges durchgemacht, ans der Erblindung und Verkleinerung des Auges resultierte. Im Alter von 2 Jahren wurde das Kind in einer hiesigen Klinik einer Augenoperation unterworfen ${ }^{1}$ ). Seit nunmehr 5 Wochen bemerkte die Mutter in dem blinden Ange des Kindes einen langsam grösser werdenden gelblichen Streifen.

Über Schmerzen in dem erblindeten Auge ist niemals geklagt worden.

Die Untersuchung des körperlich und geistig gut entwickelten Kindes ergibt, abgesehen von sehr geringen Drüsenschwellungen auf beiden Seiten des Halses, völlig normale Verhäitnisse. Wassermannsche und Pirquetsche Reaktion sind negativ. Auch in der Familienanamnese findet sich nichts irgendwie Belastendes.

Das linke Auge ist völlig gesund.

Das rechte Ange steht in Konvergenzstellung; es ist absolut blind und im ganzen ein wenig verkleinert; seine Tension ist leicht herabgesetzt; es ist völlig reizlos und zeigt absolut keine Druckempfindlichkeit.

Die Cornea ist klar und durchsichtig bis auf ein kleines strichförmiges parazentral gelegenes Leucoma adhaerens.

Die vordere Kammer ist sehr seicht und lässt bei geeigneter seitlicher Beleuchtung zahlreiche hellglänzende (Cholestearin-?) Krystalle erkennen.

1) Nach freundlicher Mitteilung von Herm Kollegen Hethey, dem ich für seine Mühe bestens danke, wurde damals ein kleines zentrales Leucoma adhaerens und im Pupillargebiet weiche, mit der Iris verwachsene Kapselschwarte festgestellt. Es wurde eine Iridektomie nach oben aussen und gleichzeitig Entfernung der Kapselschwarte nebst Linsenresten vorgenommen. Bei der Entlassung waren ophthalmoskopisch Glaskörperstränge sichtbar. (Dr. G.) 
Die Iris ist von verwaschener Struktar und graubrauner Farbe und lässt nur an einer etwas lateral excentrisch gelegenen Stelle die Gegend der völlig verwachsenen ehemaligen Pupille in Form einer seichten Vertiefung eben noch erkennen, ungefähr dem Sitz des vorher erwähnten Leulsoms entsprechend.

In der Iris befindet sich, fast genau nach aussen, ein kleines artifizielles Colobom, ans dem bei der Spiegeluntersuchung nur undentlich schwaches rotes Licht za erhalten ist. Einzelheiten des Fundus sind nicht zu erkennen. Auch ob die Linse vorhanden ist, lässt sich mit Sicherheit nicht entscheiden.

An den unteren Colobomschenkel schliesst sich unmittelbar eine gelbrötliche Neubildang von feinhöckeriger Oberfläche mit ziemlich reichlicher Vaskularisation an. Sie hat die Gestalt einer schmalen, an ihrer breitesten Stelle ungefähr $2 \mathrm{~mm}$ messenden Mondsichel, die den Kammerwinkel völlig ausfüllt und mit ihrer medialen Spitze noch ungefähr $2 \mathrm{~mm}$ über die Mittellinie nach innen ragt. Spuren einer frischen Entzündnng sind an dem Berührungsgebiete von Iris und Neubildung nirgends zu sehen.

In der freien Lücke des Coloboms sieht man bei seitlicher Beleuchtung eine graue, anscheinend gefässlose kompakte Masse sich nach unten and hinten in den Glaskörperraum verlieren.

Bei der Durchleuchtung des Auges mit der Sachsschen Lampe leuchtet die Lücke in der Iris von überall her rot auf, nur nicht, wenn man die Lampe auf die Sklera direkt unterhalb der Cornea aufsetzt.

Da es sich um eine ziemlich rasch wachsende Geschwulst in einem erblindeten Ange handelte, konnte es nicht fraglich erseheinen, dass die Therapie sick auf die Entfernung des Augapfels zu beschränken hatte. Es wurde daher am 17. III. die Enucleation des Bulbus in Äthernarkose vorgenommen; der Heilverlauf war ein glatter; nach 10 Tagen wurde das Kind aus der Klinik entlassen, nach weiteren 4 Wochen konnte schon die Glasprothese eingesetzt werden.

Der kleine Patient hat sich zum letzten Male Mitte August bei völligem Wohlsein vorgestellt.

(Dr. G.) Der Bulbus wurde nach Ciacei $0^{1}$ ) behandelt. Nach einigen Tagen wurde eine Kalotte, welche auch ein Stück des Tumors enthielt, abgetragen und mit dem Gefriermikrotom geschnitten, der Rest der Vorderhälfte in Paraffin, der Hinterabschnitt in Celloidin eingebettet.

\section{Mikroskopischer Befund.}

Medial von der Mitte besteht ein kleines Leucoma adhaerens mit Verdickung der Descemet. Die Vorderkammer enthält etwas feinkörnig geromene Massen und gequollene, mit grossem Kern versehene Zellen, welche der Irisvorderfläche aufliegen.

Die Iris liegt medial dem an sich normalen Lig. pectinatum dicht an, eine feste Verwachsung scheint nicht zu bestehen. Temporal ist die Iriswurzel durch den Tumor an das Ligament angedrängt.

Die Regenbogenhant ist auffallend reich an faserigem Bindegewebe,

1) Zentralbl. f. allg. Pathol. usw. Bd. XX. S. 385. 1909. 
namentlich auf der temporalen, dem Tumor entsprechenden Seite. Hier besteht auch eine starke zellige Infiltration, und die Gefässdurchschnitte sind viel zablreicher als medial. Die infiltrierenden Zellen sind meist einkernige Rundzellen lymphocytären Charakters, welche im ganzen unregelmässig, hie und da etwas dichter gehäuft, im Gewebe verteilt sind. Dazwischen finden sich in mässiger Menge Mastzellen und vereinzelt Plasmazellen, sowie grössere Elemente mit blasigem Kern, wohl endothelialer Herkunft. Besonders fallen sowohl in der dem Tumor anliegenden Irispartie als in der bindegewebigen Hülle der Geschwulst (siehe unten) zahlreiche typisehe ein- und zweikernige eosinophile Leukocyten anf. In der an den Tumor angrenzenden Irispartie sieht man kleine Blutungen und Häufchen von Blutpigment.

Auf der temporalen Seite des Schnittpräparates zeigt sich der rundliche, ungefähr $3 \mathrm{~mm}$ lange, ungefähr $2 \mathrm{~mm}$ breite Tumorquerschnitt (vgl. Fig 2, Taf. XIII) begrenzt vom Ciliarteil der Iris, Lig. pectinatum und Hornhauthinterfläche. Das faserige Bindegewebe der Iris, welches oberflächlich am dichtesten ist, überzieht in lockerer Anordnung die ganze Geschwulst. Nur auf diese Weise ist ein inniger Zusammenhang zwischen Tumor and Iris hergestellt.

Das Pigmentblatt der Iris ist im ganzen intakt. Temporal aber findet sich am Ciliarteil zwischen den beiden Blättern desselben ein grosser, mit feinkörniger Masse und Fibringerinnseln gefullter Hohlraum, eine Exsudationscyste. Dabei erseheint die innere Pigmentlage sehr schütter und stellenweise aufgelöst (vgl. Fig. 2, Taf. XIII).

Die Geschwulst selbst ist vollständig gefässlos. Auch in der bindegewebigen Umhüllung habe ich keine Gefässe gefunden; was klinisch so aussah, waren nur kleine streifige Blutungen (siehe unten). Der Tumor besteht ans dicht ohne nachweisbare Intercellularsubstanz aneinander gelagerten Zellen verschiedener Art, zwischen welchen lockere Bündel feiner Bindegewebsfasern verlaufen. Diese zweigen aus dem Überzug des Tumors und der Iris ab und teilen in der Geschwulst mehr weniger abgegrenzte, engere und weitere, alveolenähnliche Räume ab.

Gefrierschnitte zeigen im Tumor enorme Mengen von Krystallen. Sie haben die Form von feinen, langen Nadeln und liegen meist in dichten Büscheln zusammen. Bei Jodzusatz bleiben sie unverändert, während viele Zellen der Geschwulst, namentlich Riesenzellen tiefbraun gefärbt werden. Auch Schwefelsäure lässt sie unbeeinflusst. Nach Auflösung in Alkobol, Xylol usw. bleiben im Gewebe länglich rhombische oder spindelförmigo, oft mit bogenförmig ausgebuchteten Seiten versehene Spalten zurück.

Es dürfte sich um Fettsäurekrystalle handeln.

Ferner finden sich an verschiedenen Stellen kleine rundliche und längliche Häufchen mehr weniger veränderter roter Blutkörperchen, hier und da auch etwas Blutpigment, letzteres mehr in den Randteilen.

Die dichte Zellmasse des Tumors besteht hauptsächlich aus massenhaften Riesenzellen versehiedener Form und Grösse mit im Schnittpräparat bald am Rande ring- oder halbringförmig, bald zentral liegenden sehr zahlreichen Kernen. Letztere sind meist unregelmässig oval, saftreich mit körnchenartiger Verteilung des Chromatins und daher nicht intensiv 
gefärbt, zum Teil aber liegt das Chromatin dichter, und solche Kerne, die dann meist etwas kleiner sind, erscheinen stark gefärbt.

Es sei hier eingefügt, dass vereinzelte Riesenzellen anch in der bindegewebigen Halle des Tumors und in der oberflachlichen Irisschicht zu finden sind.

Zwischen den Riesenzellen liegen ganz dicht gedrängt unregelmässig rundliche und längliche Elemento mit meist grossem bläschenförmigen oder ovalem oder gekerbtem, seltener wurstförmig länglichem Kern, welcher, wie die Mehrzahl der Riesenzellenkerne, gewöhnlich viel Kernsaft und wenig Chromatinkörnchen entbält. Diese Zellen dürften endothelialer Natur sein.

Polynukleäre Leukocyten und kleine Lymphocyten finden sich äusserst spärlich, unzweifelhafte spindelförmige Fibroblasten habe ich nicht gesehen.

In dieser Zellmasse verlaufen die oben beschriebenen welligen, lockeren Bindegewebsfaserzüge, indem sie in unregelmässiger Weise die Schnittfläche in Felder abteilen. Die Fasern schliessen bald mehr bald weniger Zellen ein, sehr häufig füllt eine einzelne Riesenzelle einen Zwischenraum vollständig aus.

Die oben beschriebenen Krystalle liegen in ungleichmässigen aber dichten Haufen teils zwischen den Elementen, teils zu einem oder za mehreren in den Riesenzellen. An zwei Stellen findet sich je ein grosser rundlicher Haufen von Krystallen in einer amorphen Masse (Fig. 2, Taf. XIII), in welcher nur wenige kleine, runde Kerne sowie Kerntrümmer und ziemlich reichlich Reste roter Blutkörperchen sichtbar sind.

Hinter der Iris, aber nicht mit ihr verwachsen, verlanfen Stränge zarten, teils faserigen, teils mehr homogenen Bindegewebes. An diesem ist nur die oben beschriebene Pigmentblattcyste an einem Punkte adhärent, sonst befindet sich zwischen Bindegewebe und Iris überall ein leerer Zwischenraum. Auch in diesem Bindegewebe liegen Krystalle und Riesenzellen, in grosser Menge temporal, vereinzelt auch medial, während die Mitte frei ist. Die Riesenzellen enthalten hier, wie das ja an dieser Lokalität häufig zu beobachten ist, vielfach mehr weniger Pigmentkörnchen. ans dem Irispigmentblatt.

Die Linse fehlt, auch von der Kapsel ist nichts zu finden; die Zonulafasern aber erscheinen normal.

An der Pars plana des Ciliarkörpers sieht man die gewöhnlichen cyclitischen pigmentierten und unpigmentierten Epithelwucherungen in sehr ausgebildeter Weise. Die epithelialen Balken und Zotten erstrecken sich weit in das retroiridale Bindegewebe hinein. Der Ciliarkörper selbst ist nach Form und Grösse normal, zeigt auch keinen abnormen Zellgehalt.

Die Chorioidea ist stark aufgelockert, ödematös und in ungleichmässiger Weise nicht sehr dicht kleinzellig infiltriert. Auf ibr finden sich, besonders im vorderen Abschnitt, zahlreiche bindegewebige und knöcherne Auflagerungen mit Pigmentepithelwucherung.

Die Retina ist total abgelöst. Im subretinalen Raum sieht man feinkörnig geronnene Massen, aber keine Krystallspalten. Die Netzhaut zeigt die unter solchen Verhältnissen häufigen degenerativen und alten chorioretinitischen Veränderungen: Schwund der nervösen Elemente, besonders 
der Ganglienzellen - und Nervenfaserschicht, sowie der Stäbchen und Zapfen, dabei aber keine Dickenabnahme, sondern Dickenzunahme durch Gliawucherung, welche sich nicht nur im Gewebe selbst abspielt, sondern auch uber die Limitans interna hinausgeht, ferner Cystenbildungen; Neubildung und Verödung von Gefässen ohne Intimawucherung - nur an einem einzigen Gefäss findet sich zellige Wandinfiltration -, bindegewebige Adventitiaverdickung, hyaline Umwandlung and Verkalkung der Gefässwand; endlich Knoten von bindegewebsähnlicher Masse mit mehr weniger dicht pigmentierten Epithelien und andern Zellen, Produkte des gewucherten Pigmentepithels, welche bei der Ablösung der Netzhaut in dieser haften blieben. Sehr zahlreich finden sich Häufehen von Blutpigment und in deren Nähe manchmal einige randliche, lipoidhaltige Zellen.

Der Glaskörper erscheint zum Teil durch zellarmes gefässhaltiges Bindegewebe ersetzt, stellenweise finden sich in den spaltförmigen Ränmen zwischen den Netzhautfalten, welche dem Glaskörperraum entsprechen, Exsudatzellen. Vorn liegt an Stelle des Glaskörpers das oben beschriebene lockere Bindegewebe, in welches auch gefässhaltiges Gliagewebe aus der Vorderfläche der Netzhaut in ungleichmässiger Weise einstrahlt. Temporal liegt in dem Bindegewebe auf der Netzhautvorderfläche eine grosse ältere Blutung mit massenhaften Krystallen. -

Sowohl in dem Tumor als in der hinter der Iris gelegenen, Riesenzellen, Krystalle und Blutungen enthaltenden Partie lassen sich im Gefrierschnitt reichliche Massen von sudanfärbbarer Substanz darstellen. Auch in dem temporalen Iristeil sowie in der bindegewebigen Tumorhülle liegen nach Sudanfärbung zahlreiche rote, mit länglichem, gut erhaltenem Kern versehene Zellen.

Im Tumor finden sich die sudangefärbten Teile in Form von Bröckeln und feinen, bis staubförmigen Körnchen, teils in den Zellen, auch den Riesenzellen, teils zwischen ihnen. Die Verteilung ist auf dem Quersehnitt eine ungleichmässige; hier erscheinen nur einzelne Zellen rot, dort ausgedehntere Partien, vielfach schliessen die rotgefärbten Teile sudanfreie Felder ein und scheinen dann dem Verlauf der Bindegewebsfasern zu folgen.

Unter dem Polarisationsmikroskop sieht man stellenweise - ansser den Krystallen - feine Pünktchen und Krümelchen aufleuchten. Tropfen mit Achsenkreuzen sind nicht zu sehen, was vielleicht auf die Vorbehandlung zurückzuführen ist.

Nach Ciaccio) (Paraffinpräparat) lassen sich in dem Tumor sowie in der Krystalle und Riesenzellen enthaltenden Masse hinter der Iris sehr reichlich, weit mehr als nach der Anisotropie zu vermuten gewesen wäre, sudanfärbbare Körnchen nachweisen, welche, ähnlich wie im Gefrierschnitt, teils die Zellen dicht erfüllen, teils zwischen ihnen gelagert sind. Doch ist die Menge der so darstellbaren Substanz bei weitem nicht so gross wie im Gefrierschnitt. Die Iris und das den Tumor umbüllende Bindegewebe ist in den Ciacciopräparaten frei von sudanfärbbaren Elementen.

$\left.{ }^{1}\right)$ Zentralbl. f. allg. Pathol. usw. 1909.

v. Graefe's Archiv für Ophthalmologie. LXXXI. 1. 
Es handelt sich also bei den letzteren um gewöhnliches Fett, bei den im Tumor und in der Umgebung der Blutungen auftretenden sudanfärbbaren Körnchen nicht nur um Fett, sondern daneben auch um Lipoid, speziell wohl Cholesterinfettsäureester ${ }^{1}$ ).

\section{Epikrise.}

Wir finden also im wesentlichen eine alte, noch nicht abgelaufene Iridocyclochorioiditis mit Netzhautablösung. $\ddot{A}$ tiologisch ist die Entzündung höchstwahrscheinlich mit den Masern in Zusammenhang zu bringen. $\mathrm{Ob}$ sie metastatisch oder ektogen nach einer schweren Keratitis durch Sekundärinfektion entstanden war, ist nicht zu entscheiden.

Im Verlauf der Erkrankung sind auch zahlreiche kleine Blutungen aufgetreten. Diese (und ebenso die zellhaltigen Exsudate) konnten bei den stark veränderten Cirkulationsverhältnissen des Bulbus nicht in normaler Weise resorbiert werden, sie blieben liegen, es kam zu "Liposis" und „Lipoidosis" (Kaiserling) der Exsudate, wobei Fettsäuren auskrystallisierten. Die letzteren wirkten als Fremdkörper und gaben zur Bildung der Riesenzellen Veranlassung, welche wohl zum grössten Teil aus solchen endothelartigen Zellen, mit denen sie im Tumor vermischt liegen, entstanden sein dürften.

Der "Verfettungsprozess", welcher hier bei der mangelhaften Resorption zur Zersetzung unter Ausscheidung von Krystallen führt, scheint noch nicht völlig zum Stillstand gekommen zu sein; denn auch in dem lockeren Bindegewebe auf der Tumoroberfläche sowie auf diesem selbst finden sich noch verfettete Zellen und im Irisgewebe noch eine Blutung, die dem gleichen Schicksal anheimfallen dürfte. Es ist wahrscheinlich, dass auf diese Weise der Tumor noch weiter gewachsen wäre, wobei sich zwischen den Bindegewebsfasern immer neue Zellen anhäufen, die dann wieder vom Bindegewebe überzogen werden, bis die Abkapselung eine definitive wird. -

In einem ähnlichen Fall fand Helbron ${ }^{2}$ ) ausser Fettsäurenadeln namentlich massenhafte Cholesterinkrystalle. Letztere werden in der Vorderkammer bekanntlich nicht ganz selten beobachtet und auf zwei Quellen zurückgeführt: auf die Linse oder auf Blutungen und zellige

I) In einer soeben abgeschlossenen Arbeit habe ich mich mit dem Vorkommen und Verhalten der lípoiden Substanzen im Bulbus eingehend beschäftigt und möchte daher hier diese Verhältnisse nicht näher erörtern. Nur das möchte ich bemerken, dass ich fast überall, wo pathologischerweise Neutralfette vorhanden waren, auch nach Ciaccio darstellbare Lipoide gefunden habe (Keratitis, Ret. purulenta, Herde bei Anaemia pernic. und Ret. album, Sarkome der Aderhaut u. a.).

2) Zeitschr. f. Augenheilk. Bd. IV. 1900. 
Exsudate. Besonders in der älteren Literatur überwiegt die erstere Anschauung. Aber wenn auch für manche Fälle die Möglichkeit, dass das Cholesterin in der Linse gebildet und nach Perforation der Kapsel in die Vorderkammer ergossen worden sei, nicht auszuschliessen ist, muss doch betont werden, dass auch in diesen Fällen die Möglichkeit der andern Bildungsart des Cholesterins vorhanden ist, da auch hier, so weit mir die Literatur bekannt ist, wohl entzündliche Prozesse mitgespielt haben.

Wo die Linsenkapsel eröfnet war, besteht zwar theoretisch die ersterwähnte Möglichkeit; aber man braucht meines Erachtens nicht darauf zurückzugreifen, zumal die Bildung von Cholesterin (und wohl auch Cholesterinfettsäureestern) aus mangelhaft resorbierten Blutungen und Zelldepots einen in der Pathologie nicht seltenen Vorgang darstellt.

Was den Bulbus anbetrifft, so kennt man längst die cholesterinhaltigen subretinalen Exsudate bei alter Chorioiditis, welche klinisch oft das Bild des amaurotischen Katzenauges bietet (Pseudogliom), und bei der es auf der Aderhaut zur Bildung kleiner, im wesentlichen aus Cholesterinkrystallen und Riesenzellen bestehenden Knötchen kommen kann1). So hat denn auch schon Wagenmann²) in einem ähnlichen Fall wegen der im ganzen Bulbus, auch subretinal, vorhandenen Krystalle die Entstehung der letzteren aus der Linse zurückgewiesen und sie auf Blutungen usw. zurückgeführt, während Helbron $^{3}$ ) für den von ihm veröffentlichten Fall, in welchem die Kammer ganz von Cholesterin, Fettsäurekrystallen, Fibrin und Myelin ausgefüllt war, für die Cholesterinkrystalle den zuletzt angeführten Entstehungsmodus neben dem ersteren gelten lassen will. Vossius ${ }^{4}$, welcher das Auftreten von Krystallen auf der Iris im Verlauf einer Iritis klinisch beobachtete, ist geneigt, dieselben mit dem Zerfall von Hornhautpräcipitaten in Zusammenhang zu bringen; doch waren auch in diesem Fall Blutungen in der Iris aufgetreten.

Da nun so häufig die alleinige Entstehung der Krystalle aus der Linse auszuschliessen ist, so sehe ich keine Veranlassung, bei einer auch in der Pathologie anderer Organe nicht so seltenen Er-

1) Cramer u. Schultze. Arch. f. Augenheilk. Bd. XXIX. 1894. - Auch in dem von mir (Zentralbl. f. Angenheilk. 1904) beschriebenen Fall habe ich in der kleinen Aderhautgeschwulst nachträglich Krystallspalten gefunden.

2) v. Graefe"s Arch. f. Ophth. Bd. XLII, 2. 1896.

3) Zeitschr, f. Augenheilk. Bd. IV. 1900.

4) Zentralbl. f. Augenheilk. 1910. S. 266. 
scheinung für das Auge noch einen besonderen Modus anzunehmen, möchte vielmehr auch hier die Bildung des Cholesterins allein auf die in regressiver Umwandlung begriffenen Blutungen und zelligen Exsudate, deren Resorption nicht in normaler Weise vor sich gehen konnte, zurückführen. -

Besonderes Interesse bietet der vorliegende Fall in diagnostischer Beziehung. Die gelbliche Masse machte durchaus den Eindruck einer aus dem Kammerwinkel herauswachsenden Neubildung, ja man musste sogar daran denken, ob nicht eine solche vielleicht vom Ciliarkörper her unter Durchbrechung oder Abdrängung der Iriswarzel in die Kammer vorgewuchert wäre. Bei dem Vorhandensein der klinisch für Cholesterin gehaltenen Krystalle in der Vorderkammer (die bekanntlich hier im Gegensatz zu den Krystallen bei Synchisis sintillans corp. vitrei nicht ohne wesentliche Veränderungen der Umgebung entstehen) war das. Bestehen einer entzündlichen Affektion sicher. Aber daneben, bzw. als deren Ursache konnte sehr wohl ein Tumor vorhanden sein. Sogar bei einem Aderhautsarkom kann das erste auffallende Symptom eine Iritis sein, und Blutungen, aus denen die Krystalle herstammen konnten, sind ja ein bei Uvealsarkom nícht seltenes Vorkommen.

Ein Leukosarkom war bei dem kindlichen Alter des Patienten ja unwahrscheinlich. Gegen eine tuberkulöse oder syphilitische Granulationsgeschwulst sprach die Reizlosigkeit des Auges, gegen Tuberkulose ferner der negative Ausfall der Pirquetschen Reaktion. Der Tumor erinnerte mich aber sehr an einen von Dr. Kuthe klinisch beobachteten, von mir anatomisch untersuchten Fall von "Gliom der Pars ciliaris"1). Auch hier war eine gelbliche, aus dem Kammerwinkel vorwachsende Geschwulst (vgl. loc. cit. Taf. VII, Fig. A) mit entzündlichen Veränderungen (Präcipitate) in der Vorderkammer zu sehen. Es schien mir möglich, dass in dem vorliegenden Fall nur die entzündlichen Veränderungen einen höheren Grad erreicht hatten, sonst aber die Verhältnisse ähnlich lagen wie dort, und so hielt ich das Bestehen einer solchen malignen epithelialen Geschwulst des Ciliarkörpers für das wahrscheinlichste.

An einen aus Fremdkörperriesenzellen bestehenden Pseudotumor hätte allerdings bei dem Vorhandensein der Krystalle gedacht werden müssen. Man bätte dann wohl noch abwarten und den Fall weiter beobachten können; es wäre aber dann wahrscheinlich doch zu

1) Festsehr. f. J. Hirschberg. S. 127. Veit \& Co. 1905. 
Schmerzen, vielleicht zu Drucksteigerung gekommen und die Enucleation des blinden, schwer zerstörten Augapfels wäre wohl doch in absehbarer Zeit notwendig geworden.

\section{Erklärung der Abbildungen auf Taf. XIII, Fig. 1 u. 2.}

Fig. 1. Klinisches Bild des Auges.

Fig. 2. Schnitt durch die Mitte des Tumors. Vergr. $=50$. van Gieson.

$C$ Cornea (nur die innere Hälfte des Parenchyms abgebildet). - $K w$ Kammerwinkel. - - . c. Corpus ciliare. $-I$ Iris. $-B$ aus der Iris hervorgegangenes Bindegewobe, welches den Tumor gegen die Kammer zu überzieht. $-E$ locker anbaftende Exsudatzellen. - $P$ Cyste des Pigmentblatts. 


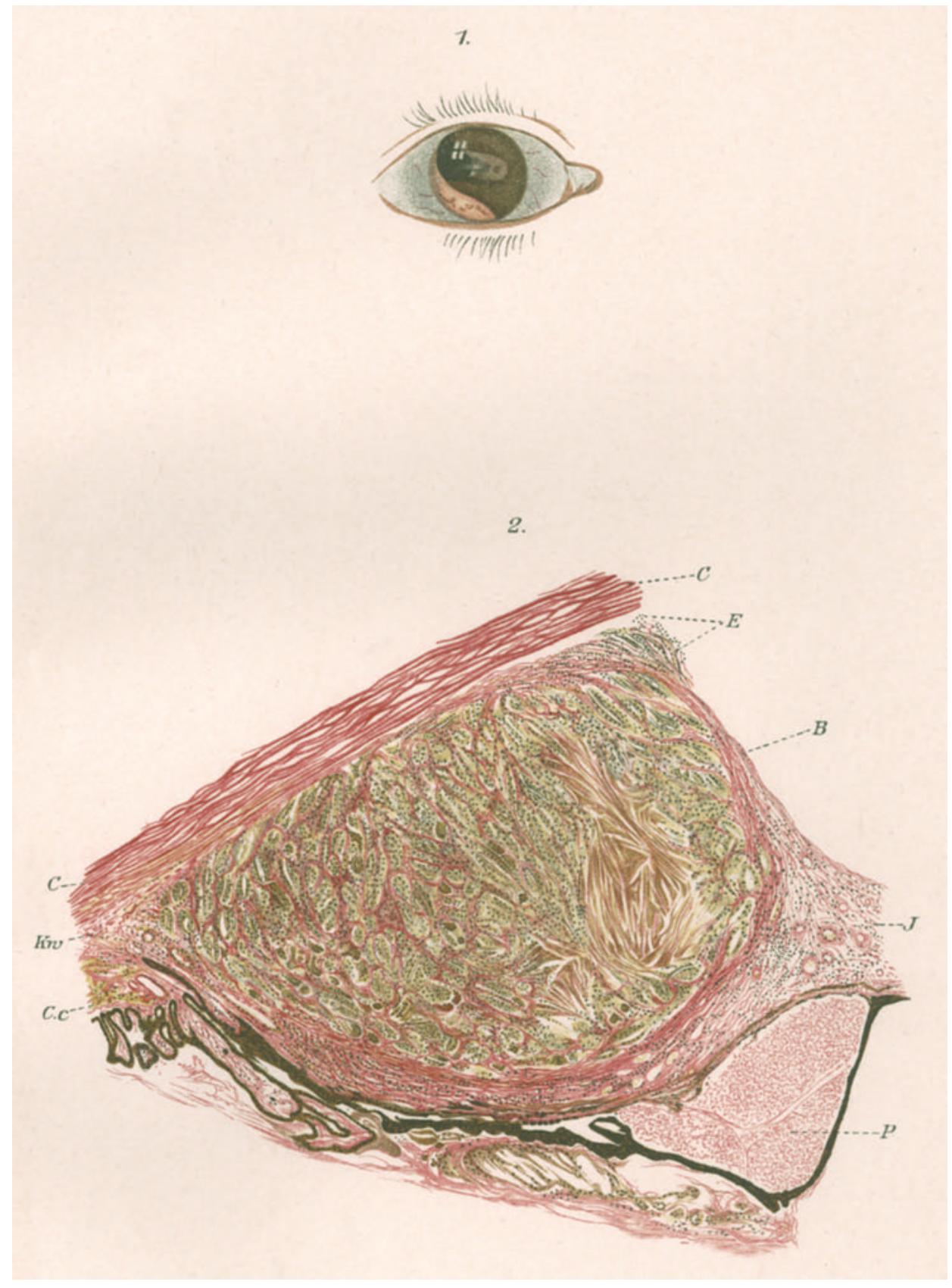

Verlag v. Wïhelm Fngelmann in Leinzig. 